\title{
Tiziano Dorandi
}

\section{Un libro dell' $A b$ initio bellorum civilium di Seneca il vecchio e il fondo latino della biblioteca della Villa dei Papiri a Ercolano}

\begin{abstract}
The recent discovery of remains of a book of the Ab initio bellorum civilium of Seneca the Elder in the PHerc. 1067 is important not only because it proves that this work was preserved in the library of the Villa des Papyri at Herculaneum, but also for its contribution to the dating of other Latin papyri of same library. Taking into account that the work of Seneca the Elder was published by his son Seneca the Young only after the death of his father (c. $39 \mathrm{CE}$ ), it is obvious that the P.Herc. 1067 was copied after this date or possibly a few years ago.

In my article, after giving an overview of the studies on the latin scrolls of the Library of Herculaneum and the research on their contribution to the palaeography of the Latin papyri, I develop some additional hypotheses that take into account the new data of P.Herc. 1067.

I then try to understand why the owners of the Herculaneum Villa in the first century CE (probably still the Pisones family) would have had interest in owning a copy of Seneca the Elder's work in their library.
\end{abstract}

\section{Premessa}

Negli ultimi decenni, i papiri latini della Biblioteca di Ercolano hanno suscitato un sempre più largo interesse che ha portato a risultati spesso innovanti e convincenti.

È stato provato che nella Villa di Ercolano non esistettero due biblioteche distinte, una greca, che corrisponderebbe grosso modo alla raccolta di libri riunita dal filosofo epicureo del I a.C. Filodemo di Gadara, e una latina sulla cui estensione e sui cui contenuti molto si è speculato. I reperti latini sono poi stati studiati nella loro globalità senza soffermarsi esclusivamente sui rotoli meglio conservati, e quindi meglio leggibili e decifrabili, trascurando gli altri in troppo cattivo stato. I progressi nelle indagini sulle tipologie grafiche latine di epoca tardorepubblicana e dei primi secoli dell'Impero, tra il I a.C. e il II d.C., hanno inoltre consentito di meglio inquadrare e classificare le scritture di quei volumina attraverso analisi dettagliate e primi abbozzi promettenti di sintesi. Infine, è stato possibile sfatare 
in maniera definitiva ipotesi attribuzionistiche attrattive, ma false, e proporne nuove confortate da dati reali affidabili quali la presenza di subscritiones.

La scoperta più importante è senza dubbio quella legata all'editio princeps del P.Herc. 1067 curata da Valeria Piano. La lettura e l'integrazione della subscriptio e lo studio accurato delle pur magre reliquie di quel rotolo hanno confermato l'intuizione di Robert Marichal che esso tramandava un libro di un Lucio Anneo e provato che quel libro apparteneva all'opera perduta di Seneca il vecchio (c. 54 a.C. - c. 39 d.C.), padre dell'uomo politico e filosofo omonimo, intitolata $A b$ initio bellorum civilium.

\section{Un'unica biblioteca greca e latina a Ercolano}

L'acquisizione che nella Villa dei papiri a Ercolano vi fosse una sola biblioteca che riuniva libri greci e libri latini è recente, che riviene a Radiciotti. ${ }^{1}$ Essa si fonda sull'analisi dell'insieme dei volumina greci e latini ercolanesi, sui dati archeologici relativi al loro ritrovamento e sull'esame storico delle fonti di cui disponiamo a proposito delle biblioteche nel mondo romano tra la fine della Repubblica e i primi tempi del principato. ${ }^{2}$

Questa realtà di fatto è del tutto normale per una biblioteca costituita in ambiente romano nella metà del I a.C. L'idea della “doppia biblioteca” nasce all'inizio dell'età imperiale nel contesto delle biblioteche pubbliche di Stato, in seno ad una scelta politica di promozione del latino, come lingua di cultura intellettuale superiore, del tutto raffrontabile al greco: il che caratterizzerà il resto dell'età romana.

In precedenza, aveva predominato l'ipotesi che nella Villa erano presenti due biblioteche: una greca e una latina e che queste due raccolte non solo erano distinte fra loro in considerazione della lingua delle opere in essi conservate, ma anche sistemate in zone differenti dell'edificio. ${ }^{3}$

1 Radiciotti (2009) i cui risultati sono ripresi e integrati da Capasso (2013a).

2 Radiciotti (2009) 113-114, da cui la citazione. Si veda anche Ammirati (2010) 33; (2015) 25.

3 Cito, a titolo di esempio, Cavallo (1984) 23-26 (= 2005, 145-146). Ma si veda ora l'onesta retractatio in Cavallo (2015) 577 n. 9, dove viene anche fatto opportunamente notare che "pur in assenza di una bipartizione tra biblioteca greca e biblioteca latina nel senso di una loro collocazione in aule distinte - tutto lascia credere a una sistemazione di libri greci e libri latini almeno in capsae o scaffali distinti, anche se in un medesimo ambiente”. 


\section{Illusioni perdute e nuove realtà}

Knut Kleve aveva insistito a lungo, dalla fine degli anni Ottanta del secolo scorso, sulla possibilità di recuperare resti di una edizione antica del De rerum natura di Lucrezio fra i rotoli latini di Ercolano. In un primo momento, lo studioso individuò tracce dei libri I, III, IV e V (per un totale di 38 versi assai malconci) in alcune scorze della cassetta CXIV dell'Officina dei Papiri Ercolanesi di Napoli. ${ }^{4}$

Kleve attribuì quei frammenti a rotoli differenti, che furono poi inventariati come P.Herc. 1829, 1830, 1831 e s.n. I, II, III. ${ }^{5}$ In un secondo momento, il medesimo studioso credette scorgere ulteriori resti del II libro del De rerum natura nel P.Herc. $395,{ }^{6}$ un rotolo che presenta con i precedenti "una forte affinità grafica" ${ }^{7}$ e che Capasso aveva già richiamato all'attenzione per provare proprio il contrario, ossia che la precedente attribuzione a Lucrezio dei restanti papiri era impossibile. $^{8}$

Kleve ha in seguito tentato a più riprese di difendere le proprie identificazioni, ${ }^{9}$ ma le critiche stringenti di Capasso, coadiuvato per gli aspetti paleografici da Radiciotti, hanno avuto definitivamente ragione della postulata presenza del De rerum natura di Lucrezio nella Biblioteca di Ercolano. ${ }^{10}$ L'ipotesi deve pertanto essere abbandonata, così come devono essere dismesse anche le ulteriori attribuzioni, suggerite dallo stesso Kleve, di altri due rotoli ercolanesi rispettivamente a Ennio e a Cecilio Stazio: il P.Herc. 21 avrebbe tramandato un libro degli Annales di Ennio ${ }^{11}$ e il P.Herc. 78 la commedia Obolostates sive Faenerator di Cecilio Stazio. ${ }^{12}$

Capasso e Radiciotti hanno insistito soprattutto su Lucrezio; le loro obiezioni e i loro argomenti valgono comunque, mutatis mutandis, anche per Ennio e per Cecilio Stazio. ${ }^{13}$

4 Kleve (1989).

5 Capasso (1989) 264-265.

6 Kleve (2007).

7 Radiciotti (2000) 367.

8 Capasso (2003).

9 Kleve (2009).

10 È sufficiente rimandare alla dettagliata rassegna redatta da Capasso (2011) 64-86.

11 Kleve (1990).

12 Kleve (1996).

13 Radiciotti (2009) 105. Si veda anche la recisa presa di posizione di Cavallo (2015) 594-595 n.

52, che parla per tutti e tre i casi di identificazioni “destituite di qualsiasi fondamento" (594). 
Se dobbiamo rinunciare a Lucrezio, Ennio e Cecilio Stazio, sicura è invece la presenza a Ercolano di almeno altri tre rotoli che tramandano libri latini non altrimenti giunti fino a noi attraverso i canali della tradizione tardoantica e medievale: un poemetto sulla battaglia di Azio di datazione e paternità dibattute (P.Herc. 817), forse un'orazione giudiziaria di età augustea (P.Herc. 1475) e infine un libro dell'Ab initio bellorum civiulium di Seneca il vecchio (P.Herc. 1067). A questi, si può aggiungere il testo trasmesso dal P.Herc. 863 di cui si conservano tracce di difficile interpretazione della subscriptio e quello del P.Herc. 395 qualora si accetti l'ipotesi (in realtà, ben difficile da dimostrare) di Beate Beer che vi fosse copiata la tragedia Tieste di Lucio Vario Rufo. ${ }^{14}$ In un solo caso, grazie alla decifrazione e alla ricostruzione della subscriptio, siamo in grado di recuperare con certezza il nome dell'autore e di restaurare il titolo dell'opera: P.Herc. 1067, Seneca il vecchio, $A b$ initio bellorum civilium.

\section{La biblioteca latina e i suoi contenuti}

Nell'ultimo inventario redatto da Del Mastro sono repertoriati 120 frammenti di papiri latini. ${ }^{15}$ Lo studioso è cosciente che un calcolo preciso di quanti fossero all'origine i rotoli dai quali derivano quei resti è impossibile, ma attraverso una analisi delle diverse tipologie dei volumina (caratteristiche paleografiche, forma dei frammenti, colore e consistenza dei materiali) egli arriva a dedurne "in via ipotetica" che "allo stato attuale i 120 frammenti latini identificati provengono da circa sessanta rotoli originari". ${ }^{16}$

Se escludiamo il Carmen de bello Actiaco, non è possibile ricavare dai restanti volumina che poche parole e frasi prive di contesto che si rivelano per lo più insufficienti per avanzare proposte di identificazione di autori e di contenuti. Il P.Herc. 1067 costituisce l'eccezione, non la regola. Eppure, anche in tempi recenti, la tentazione di scoprire nuovi testi della letteratura latina è stata grande.

Capasso ha discusso in un'ampia rassegna tutte le proposte avanzate relative al contenuto, ai generi letterari e ai probabili o possibili autori del fondo latino

14 Beer (2009). Si veda Capasso (2011) 84-85, con un caveat sull'attribuzione del P.Herc. 395 a Lucio Vario Rufo e già Ammirati (2010) 30 n. 6. Ulteriori considerazioni sul P.Herc. 395 in Capasso (2013b) 48-55.

15 Del Mastro (2005), da cui la citazione che segue (189).

16 Secondo Capasso (2013a) 38 il numero dei rotoli sarebbe un po' più alto e si eleverebbe a circa ottanta. 
ercolanese. ${ }^{17}$ Queste pagine, fatta eccezione per il paragrafo dedicato al P.Herc. 1067, che deve essere ripreso oggi alla luce dell'identificazione del suo autore con Seneca il vecchio e del contenuto con un libro della sua opera perduta $A b$ initio bellorum civilium, sono sufficienti per avere un sguardo d'insieme informato su quei materiali.

\subsection{Il Carmen de bello Actiaco (P.Herc. 817)}

Il rotolo più studiato, in ragione anche del suo discreto stato di conservazione, è il PHerc. 817. Di esso si conservano le ultime otto colonne per un totale di 63 esametri e ventisei frammenti con emistichi di lunghezza variabile di circa altri 150 versi. ${ }^{18}$ Nelle colonne superstiti, è descritta la presa di Pelusio, la situazione in Egitto all'annuncio della sconfitta navale di Azio e la conquista di Alessandria da parte delle truppe di Ottaviano. Le cause della guerra e lo svolgimento della battaglia di Azio erano narrate probabilmente nella parte iniziale del rotolo. È verosimile inoltre che il poema contasse un altro libro nel quale erano presentati la morte di Cleopatra e il successivo trionfo di Ottaviano. Di questo secondo volumen mancano, per il momento, tracce sicure. ${ }^{19}$

Quattro problemi principali, legati fra loro, hanno animato il dibattito relativo al poema: chi ne è l'autore; quale ne è la cronologia; quale la posizione del poeta rispetto ai fatti narrati e dei protagonisti dell'evento: Ottaviano, da un lato, Antonio e Cleopatra, dall'altro; le caratteristiche paleografiche del volumen.

Per lungo tempo ha dominato l'ipotesi che l'autore del Carmen fosse il poeta di epoca augustea Gaio Rabirio. ${ }^{20}$ In tempi recenti, Gigante ha cercato di confortarne l'attribuzione piuttosto a Lucio Vario Rufo, scrittore di elegie, epica e tragedie e assiduo del circolo di intellettuali che si riunivano intorno a Mecenate e che contava Virgilio, Plozio Tucca, Quintilio Varo e Orazio. ${ }^{21}$ Gigante dà particolare

17 Capasso (2011), con qualche complemento in Capasso (2013b).

18 Capasso (2011) 45-60. L'ipotesi che il PHerc. 817 sia un falso moderno, sostenuta da Brunhölzl (1998), è stata smantellata con successo da Radiciotti (2000). Si veda anche Capasso/Radiciotti (1999).

19 Janko (2008) 59-62 ha suggerito che resti di un rotolo scritto dalla medesima mano del P.Herc. 817 e quindi probabilmente appartenenti al Carmen de bello Actiaco siano da individuare nei P.Herc. 397 e 399, dei quali si conservano oggi solo di Apografi Oxoniensi. Si tratta di una ipotesi problematica i cui punti deboli sono segnalati e discussi da Capasso (2011) 58-60; (2013a) 45-48.

20 Per questa ipotesi e altre più aleatorie, si veda il regesto di Capasso (2011) 46-48.

21 Gigante (1991) 99-117 (= 1998, 57-98). 
importanza al fatto che il nome di Vario Rufo è citato al vocativo insieme con quello di Plozio, Quintilio e Virgilio, da Filodemo a più riprese in alcuni libri del suo trattato Sui vizi e le opposte virtù. Il che sarebbe una prova a favore dell'ipotesi che il filosofo epicureo avrebbe conosciuto e frequentato quei poeti e che vi potrebbe essere un qualche legame fra il Carmen de bello Actiaco e la Biblioteca greca della Villa di Ercolano. Anche la paternità di Vario Rufo non è tuttavia esente da dubbi e bene ha fatto Capasso a mantenersi su una posizione aporetica: ${ }^{22}$

[L]e poème fut vraisemblablement composé vers les dernières années du Ier siècle avant notre ère par un poète qui entendait exalter la figure e l'œuvre d'Auguste. ... En dernière analyse, nous pouvons considérer le poème comme un témoignage sur l'impact que la phase de l'histoire romaine liée à la figure de Cléopâtre a eu sur la culture et la psychologie des contemporains.

Ritornerò sulla presenza del Carmen nella biblioteca di Ercolano e sulla datazione del P.Herc. 817 dopo avere considerato i nuovi elementi che possiamo ricavare dall'edizione dei frammenti del libro di Seneca il vecchio individuato nel P.Herc. 1067.

\subsection{Il testo oratorio del P.Herc. 1475}

L'altro volumen che conserva porzioni di testo alquanto leggibili e sufficienti per trarne qualche elemento utile per l'identificazione del suo contenuto è il P.Herc. 1475.

Costabile $^{23}$ ha consolidato la suggestione di Crönert ${ }^{24}$ che il P.Herc. 1475 tramanda esigui frammenti di un'opera di natura oratoria caratterizzata dalla presenza di una terminologia giuridica “cui appartengono per esempio le parole cuiusdam ordinis o le voci manumissio e civitas, nel medesimo contesto, i verbi refero (coniug. al particolare referens) e accerso (nella scrittura libraria in luogo di arcesso), di uso tecnico nel significato di chiamare in giudizio e accusare”. Costabile dà importanza anche alla formula introduttiva ne quid con cui si apre la prima di due colonne successive in cr. 5 e 6 (fr. 5 e 6). ${ }^{25}$ Tale formula è infatti "propria di quegli ordini, con cui il pretore disponeva l'astensione da determinati atti,

22 Capasso (2011) 54, da cui la citazione.

23 Costabile (1984), da cui le citazioni che seguono (595-597).

24 Crönert (1900) 591 (= 1975, 37).

25 Il testo delle poche parole leggibili in Costabile (1984) 605, con a fronte (604), una fotografia del pezzo. 
che sono gli interdicta prohibitoria". Più rilevante gli appare comunque l'identificazione di una lex venditionis, "un patto di compravendita la cui formulazione tipica e la cui struttura funzionale" conosciamo grazie a due passi di Ulpiano e di Papiniano conservati nel Digesto. In P.Herc. 1475 cr. 6, Costabile crede che si possa ricostruire il caso che "in una compravendita, evidentemente fondiaria, possa esistere un quid sacri, come per esempio un'ara o un sacello" (egli legge in cr. 6, 2 sacri [s] i quid eriț e in 6, 3 h] abeant o abeạnt). Nel papiro, la lex venditionis segue la colonna dove è la formula proibitoria ne quid (cr. 5, 1 ne quiḍ) e precede una proposizione introdotta da un quid o quid[ nella quale "la voce mutila sti[pu]l[ potrebbe far pensare ad una stipulatio accessoria alla compravendita" in cr. 6, 3 quid (preceduto da un interpunctum) e 4 sti[..]!![.

Tutti questi suggerimenti sono fondati su scarse tracce di lettere e dovranno essere riscontrati e confermati grazie a una rinnovata lettura dell'originale con l'aiuto delle fotografie multispettrali e dei microscopi di nuova generazione.

Definitivamente da abbandonare è invece, fino da ora, l'ipotesi che si conservi la subscriptio del papiro e l'autore del discorso sia Lucio Manlio Torquato. ${ }^{26}$ Non solo la subscriptio che Costabile attribuisce al P.Herc. 1475, e che ricostruisce $L$ (uci) Mạ [nli Torqua $] t\left[i,{ }^{27}\right.$ è in realtà quella del P.Herc. 1067, ma essa va restaurata in tutt'altra maniera. ${ }^{28}$

Se il tipo di scrittura del P.Herc. 1475, una capitale libraria assai formale, orienta verso un'opera letteraria, in assenza della subscriptio e di fronte alla estrema scarsità delle tracce leggibili è impossibile pronunciarsi con sicurezza sul genere del testo e soprattutto sul suo eventuale autore.

\subsection{P.Herc. 863}

Resti di un libro (l'ottavo?) di un'opera letteraria che avrebbe raggiunto dunque una estensione considerevole sembra siano conservati nell'assai malconcio P.Herc. 863. Del Mastro ${ }^{29}$ ha provato che quel rotolo è scritto in latino e ha individuato nella seconda cornice a destra di quella che possiamo considerare l'ultima

26 Del Mastro (2005) 191-192.

27 Costabile (1984) 597-599 (il testo a 597).

28 Piano (2017a) 241-250; si confronti infra § 7.1 Tutto questo capitolo deve oggi essere rimeditato e riscritto alla luce dei risultati di Essler (2019).

29 Del Mastro (2005) 191. Più di recente, Del Mastro (2014) 137 n. 4 ha richiamato a confronto la scrittura greca del PHerc. 362 (Epic. nat. 21), datata tra il I a. C. e il I d.C. e ha ritenuto non "improbabile che la mano del papiro greco e del papiro latino sia la stessa”. Il confronto fra i due rotoli è registrato anche da Ammirati (2015) 24 n. 13. 
colonna del volumen tracce di due righi della subscriptio, che Radiciotti ha proposto di restaurare (a séguito della lettura autoptica dell'originale) - - - explici]t | - - liber] oct(avus). ${ }^{30}$

La ricostruzione delle linee può lasciare adito a dubbi vuoi per la presenza della forma verbale explicit in quel contesto e a questa epoca vuoi per la lettura oct(avus). Del Mastro, che aveva letto solo $T$ e $O$, indica le due lettere come le prime di due righi consecutivi della subscriptio e suggerisce, seppure con dubbi, che possano essere interpretate come residui del nome dell'autore e del titolo dell'opera. ${ }^{31}$

Evito, a causa della esiguità dei dati disponibili, di insistere su ulteriori proposte di identificazioni di contenuti e di autori di altri rotoli della pars Latina della biblioteca. ${ }^{32}$ Né su quelle suggerite seppure con la necessaria cautela da Bassi nel suo studio pioneristico sui rotoli latini di Ercolano ${ }^{33}$ né su quelle più recenti di Janko, ${ }^{34}$ sviluppando anche alcune suggestioni di Lindsay. ${ }^{35}$

\section{La paleografia al servizio dei contenuti della biblioteca latina di Ercolano}

Radiciotti ha fortemente insistito sulla mancanza a Roma, per un lungo periodo di tempo e almeno fino all'ellenizzazione del primo secolo a.C., di una netta separazione tra "il mondo del libro, colla sua scrittura, e quello del documento o della scritturazione della vita quotidiana” e sulla "incapacità dei Romani di definire una tipologia libraria esclusiva da riconoscere nella forma del libro-volumen della tradizione greca": ${ }^{36}$

Questa realtà è anche, a ben vedere, una possibile spiegazione sia della varietà delle scritture dei papiri latini ercolanesi, sia dell'esiguità numerica (almeno per lo stato attuale delle nostre conoscenze) dei papiri librari latini a confronto con quelli greci di Ercolano. Da un

30 Radiciotti (2009) 114.

31 Cf. Ammirati (2010) 32 e Capasso (2011) 83 n. 222.

32 Capasso (2011) 60 e Capasso (2013a) 38. Si veda già Ammirati (2010) 32-33.

33 Bassi (1926). Cf. Radiciotti (2009) 105-106 n. 7.

34 Janko (2008) 35-40, 47-48, 62-64, 69, 90, 92, 93, 95. Si tenga inoltre anche conto dei due papiri che lo studioso attribuisce al Carmen de bello Actiaco.

35 Lindsay (1890).

36 Radiciotti (2000) 371-373, da cui la citazione (373). Lo studioso si richiama a Cavallo (1989) 701-704, 708, 720-722. 
lato infatti non è ancora avvenuta, nella cultura del libro latino, una definitiva scelta a favore della scrittura capitale più rigidamente canonica (come ad esempio quella del P. Herc. 1475) e questo spiega la presenza di capitale più 'sciolta' nell'esecuzione, quale appunto quella del $P$. Herc. 817, d'altro canto nei fatti il patrimonio di libri della cultura latina è ancora esiguo ed è costituito probabilmente a Ercolano da opere latine che non avevano la possibilità di giungere fino a noi, attraverso copie tardoantiche o medievali, perché conservate appunto in una biblioteca 'chiusa' e non in una biblioteca 'pubblica'.

Tali osservazioni sono il presupposto delle importanti considerazioni che lo studioso ha avanzato sulla paleografia dei papiri latini di Ercolano ${ }^{37}$ riprendendo e approfondendo con significativi apporti personali i risultati della ricerca dalla "impostazione analitica" sul canone della capitale romana della Petronio Nicolaj, ${ }^{38}$ riproposta anche da Cavallo. ${ }^{39}$

Radiciotti classifica le scritture dei rotoli latini di Ercolano in due gruppi principali. Al primo gruppo appartengono le scritture "formali", cioè gli esempi in capitale libraria, al secondo gruppo le scritture "non formali", cioè quelle di papiri vergati in semicorsiva. All'interno dei due gruppi, vanno a loro volta individuati sottogruppi ben distinti e definiti. ${ }^{40}$

La capitale libraria delle "scritture formali", testimoniata dai P.Herc. 359, 371, $817,1059,1067,1070,1472,1475,1484,1535$ e 1558), ${ }^{41}$ è caratterizzata da "scelte formali rigorose, che selezionano un solo disegno per ciascuna delle lettere da eseguire”. Essa mostra inoltre palmari affinità con la scrittura paretaria a pennello, con una sistematica osservanza del "principio dell'effetto chiaroscurale obliquo" realizzato dunque con un calamo con taglio della punta 'alla romana' che consentiva facilmente l'alternanza di tratti pieni e tratti sottili. ${ }^{42}$

Al suo interno spicca il sottogruppo costituito dai P.Herc. 817 e P.Herc. 1067 la cui scrittura mostra peculiarità sue proprie "per giustificare le quali si è parlato di 'grecismo' grafico, costituito da alternanza di modulo tra lettere larghe e lettere strette, nonché da una generale tendenza a preferire il chiaroscuro verticale o

37 Radiciotti (1998) 355-366.

38 Petronio Nicolaj (1973).

39 Cavallo (1984) 28 (= 2005, 147-148).

40 Anche Ammirati (2010) 31-32 individua nel fondo latino di Ercolano due "raggruppamenti secondo tipologie scrittorie": uno che si esprime in volumina in una "scrittura corsiva" e l'altro in una "scrittura posata". Cf. Ammirati (2015) 24-25.

41 Radiciotti (1998) tavv. IA-VA e le forme delle lettere tracciate nella fig. I a 358. Altri frammenti, appartenenti a entrambi i gruppi, oltre a quelli elencati da Radiciotti sono stati individuati da Del Mastro (2005) e, laddove possible, classificati secondo criteri paleografici, rifacendosi comunque ancora alla inaffidabile classificazione delle scritture latine di Kleve (1994).

42 Radiciotti (1998) 357. La problematica del chiaroscuro è ora riesaminata con risultati innovanti da Cavallo/Fioretti (2014). 
quasi" e che si distingue per l'apparenza di una "maggiore scioltezza di esecuzione rispetto al modello della capitale libraria". ${ }^{43}$

Le scritture "non formali", quelle cioè che presentano varianti di lettere all'interno di un medesimo usus scribendi, sono assai frequenti e sono attestate nei P.Herc. 21, 76, 78, 90, 153, 215, 217, 219, 342, 394, 395, 396, 412, 502, 1057, 1208, 1257, 1491, 1624, 1763 (e P.Herc. 1806). ${ }^{44}$ In questo gruppo abbondano le oscillazioni nella forma delle lettere che talora sono di tipo fortemente corsivo. Anche in questo nucleo sono individuati due sottogruppi "che costituiscono i livelli rispettivamente più basso e più alto della corsività". Il che porta a distinguere fra testimoni di una "libraria corsiveggiante" (P.Herc. 1057 e 1257) e altri di "una vera e propria corsiva antica, talora inclinata a destra, forse adattata all'uso librario" (P.Herc. 215, 217 e 394). ${ }^{45}$

Se è plausibile (ma non condicio sine qua non) che tutti i rotoli in capitale libraria "formale", come i P.Herc. 871, 1067 e 1475, conservino testi letterari che riflettevano i gusti "prevalenti nell'aristocrazia romana tardorepubblicana/protoimperiale, cui appartennero i proprietari della Villa dei papiri”, più difficile è pronunciarsi sul contenuto dei volumi in scritture "non formali". Radiciotti non esclude che, messo da parte il P.Herc. 1806 estraneo al contesto della Villa, altri rotoli con scritture fortemente corsive siano da ascrivere "ad ambito non letterario" e in particolare presuppone che "l'uso della scrittura potrebbe esser stato destinato, nel periodo immediatamente precedente all'eruzione, soprattutto a quel tipo di redazione di testi a scopo documentario ed archivistico o come scritturazione di attività quotidiane. ${ }^{46}$ Ciò non ostante, è altresì probabile che alcuni rotoli copiati in scritture "non formali" e caratterizzate da un ductus che "non è fortemente corsivo e si avvicina semmai alla fisionomia di quella "scrittura di se-

43 Radiciotti (1998) 357. Si veda anche la forma delle lettere tracciate nella fig. II a 359.

44 Radiciotti (1998) 358-360. Una scelta di papiri è riprodotta nelle tav. VB-IXB. Si veda anche la forma delle lettere tracciate nella nella fig. III a pagina 360. A questi testimoni si possono aggiungere i P.Herc. 395, 1829, 1830 e 1831.

45 Radiciotti (1998) 359.

46 Radiciotti (1998) 361-363 (da cui le citazioni). Egli sottende alla sua interpretazione l'ipotesi (oggi abbandonata, infra 71) che la Villa dei Papiri venne trasformata nei primi decenni dell'impero in villa 'rustica' e ne trae la conseguente deduzione che in quel periodo la maggior parte dei volumina "sarebbe stata semplicemente destinata a costituire una biblioteca di 'conservazione' e non 'di uso', con conseguente produzione di nuovi testi” (362-363). 
conda qualità" di cui parla l'Edictum de pretïs rerum venalium, ${ }^{47}$ possano anch'essi tramandare copie di testi letterari. ${ }^{48}$

Le conseguenze che derivano da queste conclusioni sono di primaria importanza e sono da tenere in piena considerazione: ${ }^{49}$

[A]lcune scritturazioni di vita quotidiana tipiche dell'ambiente alfabetizzato, come le lettere personali, possono presentare sia una scrittura capitale degna di un libro, sia esempi di corsiva antica vera e propria. Non è dunque possibile sulla base solo dell'esame della scrittura essere del tutto certi dell'attribuzione di un testo all'ambito librario o non librario, salvo che gli elementi di spiccata calligraficità (per l'ambito librario) o di forte corsività (nell'ambito non librario) non denuncino con chiarezza il tipo di testo tràdito.

Il che vale sia per opere letterarie sia per reperti che si collocano nel "mondo del documento e cioè [nel]la produzione di testi scritti con valore giuridico”.

Alla luce di queste ricerche, si dovrà abbandonare definitivamente il tentativo di classificazione delle scritture latine ercolanesi proposto da Kleve, ${ }^{50}$ fondato su una ricostruzione dell'evoluzione della tradizione grafica maiuscola a Roma.

Secondo Kleve, la scrittura latina romana avrebbe conosciuto una prima fase corsiva (latin cursive, che egli definisce early Roman), dalla quale si sarebbe passati, attraverso uno stadio intermedio (pre classical capital), alla capitale classica (classical capital).

In realtà, la tradizione grafica latina maiuscola si espresse:

[I]n una scrittura calligrafica (capitale), dotata di diverse caratteristiche per le epigrafi e per i libri, ma anche in una forma corsiva (corsiva antica) diffusa nell'ambito dei documenti e delle scritturazioni della vita quotidiana: ma certamente il sistema alfabetico latino aveva conosciuto prima una facies posata e poi, mano a mano che si era diffuso ed era entrato profondamente nell'uso quotidiano, aveva sviluppato una tradizione corsiva. ${ }^{51}$

47 Radiciotti (1998) 363-364. Il riferimento al passo dell'Edictum a 364 n. 34.

48 Radiciotti (1998) 363-364 n. 33 richiama l'attenzione sul P.Herc. 412 nel quale Bassi (1926) 209 aveva (seppure con cautela) scorto punti di confronto con il poema di Lucrezio, senza trarne inferenze sulla sua presenza nella biblioteca di Ercolano.

49 Radiciotti (2000) 362-364 (da cui le citazioni), 370.

50 Kleve (1994).

51 Radiciotti (2008). Citazione da 55-56. Le critiche di Radiciotti sono ribadite da Ammirati (2010) 30-31 e Ammirati (2015) 24-25, che nega ogni tentativo di applicare criteri cronologicoevolutivi alle varietà grafiche testimoniate dai papiri ercolanesi. Cf. Fioretti (2014) 41 n. 46. 


\section{Per una cronologia del fondo librario latino a Ercolano}

Radiciotti ha rinnovato anche lo studio della cronologia relativa della formazione della biblioteca latina ercolanese attraverso l'analisi comparata delle scritture dei papiri e dei testi in essi trascritti. Il quadro che ne risulta "è univoco" - scrive Radiciotti - "le indicazioni del nome di Augusto in alcuni papiri e, d'altro canto, l'esame analitico delle scritture latine attestate forniscono la stessa indicazione cronologica: i papiri latini risalgono al periodo fra la tarda repubblica e l'età augustea". ${ }^{52}$ La nascita del fondo più antico di quella biblioteca è pertanto "coeva al periodo della attività ercolanese di Filodemo”. E anche se è “pur vero che i testi in questione potrebbero esser copie di antigrafi del primo secolo avanti Cristo ... il fatto che la villa non fosse più un centro culturale attivo negli anni precedenti l'eruzione si somma ad altri indizi, per farci ritenere che i volumina latini di Ercolano di più alta qualità formale devono risalire piuttosto al tardo primo secolo avanti Cristo". 53

Questa cronologia è più precisa di quella comunemente fornita dagli studi di ampio respiro sulla paleografia dei papiri latini, che insistono piuttosto su un arco temporale che ha il limite estremo nel 79 d.C.; ${ }^{54}$ essa ha in più anche il vantaggio di escludere fuorvianti confronti dei rotoli di Ercolano "con materiali papiracei latini rinvenuti in Oriente e risalenti ad età flavia”. ${ }^{55}$

Nella sua indagine, Radiciotti ha dato grande importanza specialmente a due indizi. In primo luogo, egli ha insistito sulle affinità grafiche fra i rotoli di Ercolano e i più antichi volumina letterari latini recuperati in Oriente, che datano tra il I a.C. e il II-III d.C. Il confronto si rivelerebbe efficace in quanto "tutta la più antica produzione latina in Oriente deve essere concepita come strettamente affine a quella occidentale, perché frutto del puro ‘trapianto' della scrittura latina al seguito dell'occupazione militare romana dell'Egitto e dei regni ellenistici orientali". In secondo luogo, egli ha richiamato l'attenzione sulla presenza in Roma e in Campania, tra la repubblica e il principato, di grammatici e retori bi-

52 Radiciotti (2009) 107 (da cui la citazione che segue) e già, con maggiori dettagli, Radiciotti (1998) 365-370. Per le indicazioni del nome di Augusto, Radiciotti (2009) 107 n. 2 rinvia a P.Herc. 1067 fr. 10 l. 3 nell'edizione di Costabile (1984). Questo elemento deve ora essere valutato altrimenti dopo l'attribuzione del rotolo a Seneca il vecchio.

53 Radiciotti (1998) 365.

54 Con un rimando a Seider (1978) 34-36 (nn² 2, 4 e tavv. II-III).

55 Radiciotti (2009) 107. 
lingui che formarono "un ambiente culturale bilingue, intessuto di curiosità filosofiche epicuree, di interessi poetici e storici, di conservazione di libri" comparabile a quello che ritroviamo nella Villa di Ercolano. ${ }^{56}$

Il fatto che nei reperti orientali sia attestata una più larga categoria di scritture di "seconda qualità" rispetto a quelle della biblioteca di Ercolano dipende probabilmente dalle differenti situazioni storico-sociali e culturali legate alla produzione di quei materiali in due mondi e ambienti diversi.

Anche l'apparizione, già in epoca assai antica e molto "prima dell'affermazione sistematica del libro in forma di codice", di forme "onciali" inserite nel contesto delle scritture di "seconda qualità", va interpretata come prova ulteriore che la documentazione ercolanese risale a un'epoca più antica di quella a ridosso del 79 d.C. e deve essere considerata, per la maggior parte, "paragonabile per epoca ai prodotti librari greci di età filodemea od immediatamente posteriore". ${ }^{57}$

\section{Nuova luce dal P.Herc. 1067}

Il momento è venuto di rivedere queste acquisizioni alla luce dell'attribuzione del testo trasmesso dal P.Herc. 1067 all'Ab initio bellorum civilium di Seneca il vecchio.

Siamo di fronte a una scoperta fondamentale per molte più ragioni di quante possano apparire a prima vista e di quante siano state finora indicate.

Non ho bisogno di insistere sull'edizione, sul contenuto e sull'attribuzione del rotolo già presentati in maniera eccellente dalla Piano. ${ }^{58} \mathrm{Mi}$ soffermo invece, in particolare, sui i nuovi elementi che ne scaturiscono e che rimettono in discussione un aspetto almeno della datazione dei papiri latini di Ercolano sulla base di argomenti più solidi che non siano esclusivamente quelli delle caratteristiche paleografiche.

Sapevamo da un passo della cosiddetta Vita patris di Seneca il giovane che suo padre aveva lavorato fino alla propria morte (c. 39 d.C.) a un'opera sulla storia recente di Roma (historiae ab initio bellorum civilium), rimasta inedita (Appendix T1)..$^{59}$

56 Radiciotti (1998) 365-366.

57 Radiciotti (1998) 370.

58 Piano (2017a) e Ead., in questo volume, supra 31-50.

59 Condivido l'ipotesi, riproposta da Sussmann (1978) 144 (con ulteriore bibliografia nella n. 23; si veda anche il contributo dello stesso Sussman infra 143-146), che il passo in questione non 
A questa opera venivano di solito riportati due frammenti: uno citato da Svetonio sulla morte di Tiberio e uno da Lattanzio relativo alla concezione storiografica dell'autore. ${ }^{60}$ Poiché Svetonio e Lattanzio si riferiscono a "Seneca" senza altra specificazione, alcuni studiosi avevano espresso dubbi sulla loro attribuzione a Seneca il vecchio e avevano supposto che l'autore ne fosse piuttosto il figlio omonimo. ${ }^{61}$

La nuova lettura e la conseguente ricostruzione della subscriptio del P.Herc. 1067 rafforza l'ipotesi che quei due frammenti derivano dall' $A b$ initio bellorum civilium di Seneca il vecchio, un'opera che dovette aver conosciuto una qualche diffusione in certi ambienti culturali e forse anche una discreta sopravvivenza.

L'identificazione del testo con un libro dell' $A b$ initio bellorum civilium, la sua attribuzione a Seneca il vecchio e la sua 'pubblicazione' postuma tra la fine gli anni Trenta e gli inizi degli anni Quaranta del I d.C. sono elementi che devono essere ora riletti in relazione con la presenza a Ercolano del P.Herc. 1067 e di conseguenza con la cronologia tradizionale dei volumina latini della biblioteca della Villa scritti in capitale "formale" o in "scrittura posata".

\subsection{Considerazioni sulla subscriptio del PHerc. 1067}

Per cominciare vorrei presentare qualche osservazione complementare sulla subscriptio del P.Herc. 1067 e sulla ricostruzione che ne è stata proposta.

Il restauro della Piano ha destituito di ogni fondamento l'ipotesi che il P.Herc. 1067 tramandasse una oratio in Senatu habita ante principem ${ }^{62}$ e ha provato anche che quel rotolo trasmette frammenti di un libro dell' $A b$ initio bellorum civilium di Seneca il vecchio. ${ }^{63}$

Piano propone la seguente ricostruzione della subscriptio:

$$
\begin{aligned}
& L[\cdot] \text { Annae }[i \cdot \text { Senec }] \text { ae } \\
& A b \text { • innițịo } \cdot \text { b }[\text { ello]rum [ }[\text { civilium] } \\
& \text { [Historiae] }
\end{aligned}
$$

deriva da una 'biografia' di Seneca il vecchio, ma dalla premessa che il figlio avrebbe apposto alla sua edizione postuma dell' $A b$ initio bellorum civiulium del padre.

60 Suet. Tib. 73.2 (= FRHist 74 F 1) e Lact. inst. 7.15 .14 (= FRHist 74 F 2).

61 Si veda Canfora (2015) 138-213.

62 Piano (2017a) 241-249.

63 La presenza del nome di Seneca, già intuita da Robert Marichal, era rimasta ignota perché registrata solo nei documenti inediti del suo archivio privato. Ne avevo avuto comunicazione orale da parte dello studioso già agli inizi degli anni Novanta del secolo scorso. Si veda Dorandi (2017b) 23. 
Le lettere ae di Senecae e ru di biello]rum sono recuperate sistemando due sovrapposti. Il papiro è rotto dopo il secondo rigo.

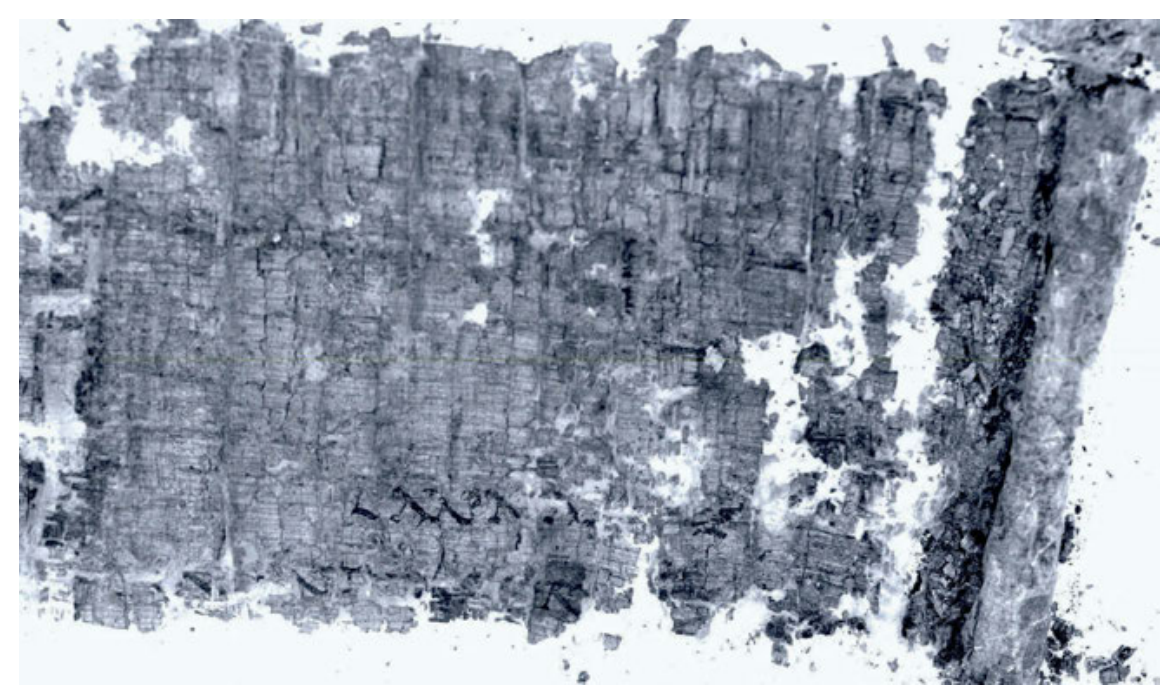

Fig. 7: PHerc. 1067, subscriptio. ( Biblioteca Nazionale di Napoli - Brigham Young University, Provo, USA

Il punto critico è costituito dal terzo rigo. La Piano propone di integrarvi, seppure exempli gratia, [Historiae] appoggiandosi sulla testimonianza del De vita patris di Seneca figlio: quisquis legisset eius historias ab initio bellorum civilium. In alternativa, essa suggerisce [Liber] o [Libri]. ${ }^{64}$

Personalmente eviterei sia Historiae sia [Liber] o [Libri]. Che l'opera fosse intitolata Historiae ab initio bellorum civilium è possibile, ma non trova una conferma cogente nella subscriptio del P.Herc. 1067, dove tra l'altro il sostantivo Historiae apparirebbe posposto rispetto alla testimonianza del De vita patris, con una anomala inversione sintattica. Quanto a [Liber] o [Libri], come parte integrante del titolo, mancano invece, a quanto sappia, paralleli cogenti. ${ }^{65}$ Penso per-

64 Piano (2017a) 241-246.

65 Non è inutile ribadire che la presenza del sostantivo liber nelle soscrizioni di codici di Livio e di Tacito non fa parte integrante del titolo originale, ma del colofone interno che assicurava il passaggio al libro successivo quando l'opere vennero trasferite da rotoli a codici. Così per esempio, per Livio, nel manoscritto Paris, Bibliothèque Nationale de France, Lat. 5730 fol. 225v: Titi 
tanto che il titolo dell'opera di Seneca il vecchio, stando almeno alla testimonianza ercolanese, fosse $A b$ initio bellorum civilium sul modello dell' $A b$ urbe condita di Tito Livio e poi dell'Ab excessu divi Augusti di Tacito nonché dell'A fine Aufidii Bassi di Plinio il Vecchio. ${ }^{66}$

A parte questo, richiamo l'attenzione sul fatto che nell'attuale ricostruzione della subscriptio del P.Herc. 1067 non c'è traccia del numero del libro dell'opera di Seneca copiato su quel rotolo. Appare evidente che un'opera che narrava la storia di Roma dagli inizi delle guerre civili (comunque si interpreti l'ab initio del titolo e la frase unde primum veritas retro abiit della testimonianza del De vita patris) e che arrivava all'avanzata età augustea e forse fino al principato di Tiberio († 16 marzo 37 d.C.), il cui o nome si legge in cr. 5 pz. II sov. 1 1. 8: Tiberius (preceduto da un interpunctum), ${ }^{67}$ si estendesse per più libri.

Il numerale poteva seguire, sullo stesso rigo, civilium oppure essere sistemato, centrato, nel terzo rigo. Suggerirei dunque: ${ }^{68}$

$A b \underline{b} \cdot \underline{i n t i t ̦ i o ~} \cdot b \underline{b}[$ ello]rum $[\cdot \operatorname{civilium}(x)]$

oppure

$A b \underline{b} \cdot \underline{\text { intịtịọ }} \cdot$ ḅ[ello]rum $[\cdot$ civilium $]$ $[(x)]$.

In entrambi i casi, $(x)$ indica il numero del libro, che rimane ignoto.

A priori, e in via del tutto ipotetica, si potrebbe pensare anche a un'altra eventualità e cioè che il numerale vi mancasse perché il P.Herc. 1067 non trasmetteva un unico libro dell' $A b$ initio bellorum civilium, ma piuttosto una scelta di estratti da diversi libri. I troppo magri frammenti e la completa ignoranza sul contenuto dell'opera invitano tuttavia a restare estremamente prudenti, anche se un sia pur labile indizio che potrebbe confortare questa ipotesi è forse conservato.

In cr. 5 pz. I, nel secondo intercolumnio all'altezza del 1.9 di scrittura, Piano ha individuato chiari "segni che sporgono a destra in un vistoso spazio interco-

Livi / ab urbe condita liber XXV explic(it) incipit liber XXVI feliciter e nel Wien, Österreichische Nationalbibliothek Lat. 15 fol. 193v Titi Livi ab urbe condita liber XLV explic(it) incipit liber XLVI feliciter. Altri dettagli in Oliver (1951) 238. Per Tacito, si vedano gli esempi dal manoscritto Firenze, Biblioteca Medicea Laureanziana 68.1 registrati da Oliver (1951) 235.

66 Quest'ultima testimoniata dallo stesso Plin. nat praef. 20 (=FRHist 80 T 5) e da Plin. epist. 3.5.6 (= FRHist 80 T 5 1). Si veda Aufidius Bassus, FRHist 78 T 3ab.

67 Piano (2017a) 236-237.

68 In entrambi i casi, l'aggiunta di liber prima del numerale è verosimile, ma non necessaria. 
lonnare, non riconducibili ad una plausibile stringa di testo". Il tratteggio dei segni assomiglia a una $M$, ma in realtà si tratta di "una sequenza di linee che disegnano forme triangolari, realizzate in modo continuo, senza spazi tra di loro". Poiché è senza dubbio da escludere che si tratti di una "stringa di testo, sembra verosimile intenderli come parte di un elemento ornamentale apposto con la finalità di distinguere due sezioni di testo". ${ }^{69}$

Questa proposta mi sembra plausibile. Resta tuttavia da chiedersi (e purtroppo una risposta è allo stato attuale impossibile) se quell'elemento ornamentale (e eventuali altri non più visibili) distinguesse "sezioni di testo" (capitoli?) di un libro oppure estratti di più libri riuniti insieme. Tentare di dire di più sarebbe controproducente e azzardato. ${ }^{70}$

La medesima cautela deve essere applicata infine anche relativamente alla questione se il P.Herc. 1067 fosse l'unico libro-volumen dell'Ab initio bellorum civilium presente nella biblioteca della Villa di Ercolano.

Queste osservazioni non mettono bene inteso in dubbio che la ricostruzione del titolo $A b$ initio bellorum civilium sia corretta e più aderente alle tracce di un titolo che riporti all'altra opera di Seneca il vecchio: Oratorum et rhetorum sententiae divisiones colores. ${ }^{71}$

\subsection{Conseguenze per la cronologia del fondo antico della biblioteca latina}

Lasciato da parte il titolo, vengo all'aspetto che mi appare più ricco di conseguenze. Dal punto di vista paleografico, Radiciotti aveva collocato il P.Herc. 1067, insieme con il P.Herc. 817, nel sottogruppo delle scritture latine di Ercolano in capitale libraria "formale" che si distingue per una "maggiore scioltezza di esecuzione rispetto al modello della capitale libraria" e che "presenta alcune caratteristiche sui generis, per giustificare le quali si è parlato di 'grecismo' grafico, costituito da alternanza di modulo tra lettere larghe e lettere strette, nonché da

69 Piano (2017a) 185-186, da cui le citazioni.

70 Piano (2017a) 186 richiama la presenza di una decorazione analoga (una serpentina di $S$ ) nel P.Qasr Ibrîm 40 (= ChLA XLII 1237), frammento latino databile tra I a.C. e I d.C. nel quale "la cornice decorativa sembra nello spazio non scritto destinato al margine superiore”. Preferisco invece non tenere conto (per ragioni cronologiche) dell'altro parallello con il P.Monts.Roca I, frammento da codice papiraceo del IV d.C., che tramanda le Catilinarie di Cicerone distinte fra loro da "linee ondulate convergenti ... disposte su più righi".

71 Piano (2017a) 246-249 e in particolare 246 n. 147. 
una generale tendenza a preferire il chiaroscuro verticale o quasi”. ${ }^{72}$ Il P.Herc. 817 è datato intorno alla fine del I a.C.; ${ }^{73}$ allo stesso periodo è stato di ricondotto anche il P.Herc. 1067.

L'attribuzione del P.Herc. 1067 all'Ab initio bellorum civilium di Seneca il vecchio rimette ora in discussione questa acquisizione. In particolare, non è più possibile accettare in toto la proposta che il nucleo più antico dei rotoli latini, ai quali sono ricondotti il P.Herc. 1067 e il P.Herc. 817, vergati in capitale "formale" o in "scrittura posata” sia da collocare nel "periodo fra la tarda repubblica e l'età augustea". ${ }^{74}$

La presenza del P.Herc. 1067 nella biblioteca di Ercolano ha come presupposto incontestabile, se non la 'pubblicazione' a cura di Seneca figlio dopo la morte del padre di quel libro dell' $A b$ initio bellorum civilium, almeno l'esistenza di una sua redazione scritta. Il che sposta di conseguenza il momento della copia del P.Herc. 1067 e quindi la datazione del rotolo tra la fine degli anni Trenta e gli inizi degli anni Quaranta del I secolo d.C.

La cronologia della composizione dell' $A b$ initio bellorum civilium risale con buona probabilità agli ultimi anni del principato di Tiberio († 16 marzo 37) o agli inizi di quello di Caligola (18 marzo 37 - 24 gennaio 41). ${ }^{75}$ Quanto alla sua 'pubblicazione' postuma da parte del figlio, essa resta controversa al punto da essere stata addirittura negata da alcuni studiosi. ${ }^{76}$

72 Radiciotti (1998) 357. Cf. Ammirati (2010) 32: “sotto il profilo dell'elaborazione è verosimile collocare la scrittura di questo papiro [sc. P.Herc. 1067] tra quella di P.Herc. 1059 e 1475, e quella di P.Herc. 817". Piano (2017a) 180 richiama l'attenzione anche sul più tardo P.Berol. inv. 11596 recto (secondo quarto del II d.C.) su cui Ammirati (2015) 30, 72 (e tav. XVII) e già Ammirati (2010) 38, 39-40 (con la tav. 2).

73 Radiciotti (1998) 367 e Radiciotti (2000) 361-362 n. 41 "scritto verso la metà del terzo decennio a.C.”. Cf. Capasso (2013a) 40 e Fioretti (2014) 35 n. 25. Per Cavallo (2015) 592, la scrittura del P.Herc. 817 "una capitale latina piuttosto fluida e connotata da una certa instabilità di tracciati conforta una datazione al I a.C., ma la cautela è d'obbligo". Altre proposte di datazione meno convincenti che arrivano fino all'età dei Flavii, registra Capasso (2011) 46-55.

74 Radiciotti (2009) 107 ripreso da Fioretti (2014) 42 n. 48.

75 Canfora (2015) 139, 208 suggerisce che Seneca ne avesse cominciato la redazione alla fine del principato di Tiberio e che il figlio l'avesse pubblicata postuma nei primi anni di Caligola, in un momento in cui il princeps aveva mostrato una apertura "verso la storiografia 'repubblicaneggiante' su cui si era abbattuta la censura (prima con Augusto poi con Tiberio)”. Si veda anche 139, "forse il vecchio si era messo a scrivere quando era avanti negli anni nell'ultimo tempo del principato di Tiberio".

76 Si veda in particolare Klotz (1901) 441-442 e Griffin (1972) 10. Status quaestionis in Levick in FRHist I 506-507, che non esclude una 'pubblicazione' anche se qualche anno più tardi e cioè dopo il rientro di Seneca figlio dall'esilio nel 49 d.C. 
Per il mio discorso la questione della 'pubblicazione' o meno dell'opera non ha una importanza cruciale. Il P.Herc. 1067 poté infatti esser stato copiato a uso 'privato' prima della eventuale 'pubblicazione' sull'esemplare personale di Seneca il vecchio su richiesta dell'allora proprietario della Villa, ${ }^{77}$ interessato al suo contenuto per ragioni (intellettuali, ideologiche o politiche) che purtroppo ci sfuggono, ma probabilmente e pur sempre nel rispetto della "prudenza ... comprensibile" che spinse l'autore a non diffondere in pubblico la sua opera storiografica in momenti pericolosi. ${ }^{78}$

Essere più precisi sulla cronologia sia pure relativa è impossibile vuoi in ragione delle difficoltà legate a datare papiri letterari latini (e greci) sulla base delle sole caratteristiche paleografiche vuoi delle incertezza del momento in cui Seneca il vecchio iniziò la composizione dell'Ab initio bellorum civilium. ${ }^{79}$ Né troviamo un aiuto in elementi interni quali la presenza nei frustuli del PHerc. 1067 del nome di Tiberius e, a più riprese, di quello di Augustus nonché di quello di Quintus Haterius (cr. 2 pz. I sov. 6 1. 5), uno dei più famosi oratori dell'epoca augustea. Troppo poco e in contesti sempre molto lacunosi perché se ne possano dedurre dati sicuri per definire ulteriormente la data dell' $A b$ initio bellorum civilium e ancora meno di quel libro incerto.

\subsection{Breve ritorno sul P.Herc. 817}

Un discorso a parte richiede il P.Herc. 817 vergato in un linguaggio grafico affine (anche se non identico) a quello del P.Herc. 1067.

Anche se le affinità delle caratteristiche paleografiche dei due rotoli possono spingere a avanzare altresì la cronologia del P.Herc. 817, sarebbe azzardato (se non inopportuno) trarre da questi soli elementi la conseguenza che la composizione del Carmen de bello Actiaco sia coeva alla copia del rotolo che lo tramanda.

77 Sono convinto che la Villa di Ercolano, fatta costruire da un membro della famiglia dei Pisoni, anche se forse non da Lucio Calpurnio Pisone Cesonino, patronus di Filodemo, rimase sempre proprietà della gens Calpurnia (Dorandi 2017a).

78 Canfora (2015) 140.

79 Per questo ultimo aspetto, valgano le considerazioni di Cavallo (2013) 1, che è lecito allargare anche alla produzione latina: "Assegnare datazioni ai papiri greci letterari, normalmente privi di qualsiasi elemento cronologico oggettivo, è operazione talora assai difficile. I criteri archeologici, bibliologici paleografici correntemente adoperati - soprattutto, nel caso di questi ultimi, quando manchino riscontri precisi con scritture documentarie datate - non sempre si dimostrano di peso tale da dare risultati soddisfacenti, sicché non sono poche le datazioni assegnate che restano incerte o aleatorie". 
Niente impedisce infatti a priori che vi sia un décalage fra la data effettiva di redazione del poema (oggi generalmente colloca alla fine del I a.C.) ${ }^{80}$ e quella della confezione P.Herc. 817, in questo caso copiato su un più antico esemplare in un'epoca e in un ambiente vicini a quelli del P.Herc. 1067.

Se queste considerazioni sono valide, potremmo addirittura suggerire di scorgere nel P.Herc. 817 e nel PHerc. 1067 due esempi tangibili della pratica comune alla società romana, fra tarda repubblica e inizio del principato, di una diffusione di libri "entro una certa fascia di lettori mediante esemplari prodotti ... privatamente ma che ... potevano dare luogo ad altre copie per doni scambi e richieste". ${ }^{81}$

\section{Tracce di vita culturale della Villa nella prima metà del I d.C.?}

Possiamo a questo punto chiederci se l'attribuzione del P.Herc. 1067 all'opera di Seneca il vecchio $A b$ initio bellorum civilium e la nuova cronologia ulteriori elementi utili per definire le sorti della biblioteca della Villa di Ercolano in epoca postfilodemea, tra la fine del I a.C. e i decenni centrali dei I d.C.

Rispondere a questa domanda non è semplice e il rischio di sopravvalutare $i$ dati di cui disponiamo è sempre presente con tutte le sue insidie.

Piano $^{82}$ ritiene che il libro tramandato dal P.Herc. 1067 era "strettamente connesso con le vicende vissute, in maniera diretta, dalla stessa aristocrazia romana che frequentava la Villa dei Pisoni" e che i dati che si ricavano dai frammenti di quel rotolo "dimostrano, in modo inequivocabile, che la domus, e con essa la sua biblioteca, era ancora caratterizzata da una grande vitalità intellettuale durante tutta la prima metà del I secolo, e dunque fino a ben poco prima dell'eruzione”. Poiché Seneca compose la sua opera tra gli anni Trenta e Quaranta se ne può altresì dedurre che la Villa "durante tutta la metà del I d.C. vide accrescere il suo patrimonio librario con opere filosofiche greche, affiancate da opere latine di altro genere, ma che insieme alla pars philosophica contribuiscono a delineare uno spaccato sempre più preciso e verosimile della vita sociale, politica e culturale della Villa dei Papiri”.

\footnotetext{
80 Supra $55-56$.

81 Cavallo (2015) 596. Con un rimando (nella n. 54) a Starr (1987) 213-223.

82 Piano (2017a) 249-250, da cui le citazioni che seguono.
} 
Non c'è alcun dubbio che questa lettura è più coerente e verosimile di quella avanzata anni addietro, limitatamente al solo fondo latino della biblioteca, da Radiciotti perché compromessa dall'accettazione dell'ipotesi di una riconversione agricola della Villa nel I d.C.: ${ }^{83}$

[I]l fatto che la villa non fosse più un centro culturale attivo negli anni precedenti l'eruzione si somma ad altri indizi, per farci ritenere che i volumina latini di Ercolano di più alta qualità formale devono risalire piuttosto al tardo primo secolo avanti Cristo.

L'ipotesi di una trasformazione in villa rustica ${ }^{84}$ è da scartare in maniera definitiva alla luce in particolare dei recenti scavi in situ che hanno mostrato che al momento dell'eruzione del Vesuvio del 79 d.C. le decorazioni pittoriche parietali della basis Villae erano in restauro. ${ }^{85}$

Mi sia consentito tuttavia esprimere una punta di scetticismo anche a proposito della lettura, almeno a mio parere, un po' troppo ottimistica della Piano e suggerire qualche pista parallela.

Senza mettere per niente in discussione tutta l'importanza della scoperta del libro di Seneca il vecchio, non riesco a scorgere in questa testimonianza e in quella della copia apparentemente coeva del P.Herc. 817, una prova determinante che la Villa "durante tutta la metà del I secolo d.C. vide accrescere il suo patrimonio librario con opere filosofiche greche, affiancate da opere latine di altro genere".

Innanzitutto, le considerazioni che ho esposto sulle conseguenze che la presenza di un libro dell' $A b$ initio bellorum civilium di Seneca il vecchio a Ercolano ha sulla cronologia relativa di una parte almeno dei rotoli latini della Biblioteca devono mettere in guardia anche sull'attribuzione, su basi esclusivamente paleografiche, di un manipolo di papiri greci a tutta la prima metà del I d.C.

Nella realtà della biblioteca di Ercolano, distinguere tra reperti della fine del I a. C. e altri dell'inizi o della prima metà del I d.C. può rivelarsi un esercizio pericoloso, che è bene praticare con la più grande moderazione. ${ }^{86}$

Piano rileva infine, a ragione, che il libro di Seneca è "strettamente connesso con le vicende vissute, in maniera diretta, dalla stessa aristocrazia romana che frequentava la Villa dei Pisoni" e che contribuisce a delinearne "uno spaccato sempre più preciso e verosimile della vita sociale, politica e culturale".

83 Radiciotti (1998) 365.

84 Wójcik (1986) 37.

85 Guidobaldi/Esposito (2009) 343-352 e Del Mastro (2010) 64-65.

86 Cf. Capasso (2013a) 35-36. 
Integrerei queste affermazioni con qualche considerazione relativa anche alla presenza forse non fortuita nella Villa di Ercolano, in momenti non troppo lontani fra loro, dell'esemplare di almeno un libro dell' $A b$ initio bellorum civilium di Seneca il vecchio e di quello di almeno un libro del Carmen de bello Actiaco. ${ }^{87}$

Simili testi oltre che per motivi letterari e intellettuali poterono infatti attirare gli interessi dei proprietari della Villa o di chi ne frequentava la biblioteca anche per il messaggio ideologico e politico legato al loro contenuto.

In ogni modo, una attenzione particolare fu in quei momenti riservata al poema trasmesso dal P.Herc. 817. Lo provano i numerosi interventi 'extratestuali' peculiari a quel rotolo rivelati dalla Scappaticcio: ${ }^{88}$ interpuncta, I longa, segni obliqui alla fine dei versi, diplai e apices scritti talora di altra mano e quindi indizi evidenti di una utilizzazione diacronica del rotolo.

La studiosa ha messo in relazione fra loro, in particolare, i segni obliqui alla fine dei versi e gli apices e indica in essi un sistema che avrebbe potuto segnalare, all'interno degli esametri, delle "cesure non tanto testuali, quanto piuttosto ritmiche”. ${ }^{89}$ I due segni appaiono aggiunte seriori di persona diversa dallo scriba che ha copiato il testo. I tratti obliqui "probabilmente vennero apposti per segnalare momenti in cui si dovesse arrestare la voce o regolare l'intonazione con cui cantare quei determinati esametri, concentrando la propria enfasi su elementi ben precisi”. Quanto agli apices, ${ }^{90}$ essi sono indicazioni apposte da una persona con il "chiaro intento di indicare l'esatta pronuncia di parole che probabilmente non le erano particolarmente note" e che pertanto "non aveva una conoscenza perfetta della lingua latina ... qualcuno che non aveva il latino quale lingua nativa". ${ }^{91}$ Il tutto porta la studiosa a supporre che almeno gli apices "possano avere un'evidente connessione con la lettura - direi, con la 'recitazione' - che era fatta

87 Scrivo in entrambi i casi "di almeno un libro" tenendo conto della realtà attuale e ben cosciente che questi dati potranno essere affinati o corretti da successive scoperte di nuovi rotoli o dallo svolgimento o lettura di altri.

88 Scappaticcio (2008).

89 Scappaticcio (2008) 240-245. Citazioni da 240 e 245.

90 Scappaticcio (2008) 240 utilizza convenzionalmente il termine apex (preso in prestito dall'epigrafia) “per indicare l'accento acuto apposto - per lo più sopra vocali - all'interno delle testimonianze papiracee”. Apices sono presenti anche nel P.Herc. 1067, Piano (2017a) 186. Ulteriori considerazioni in Scappaticcio (2012).

91 Scappaticcio (2008) 245-246. Un intervento per certi aspetti simile, ma su un testo greco (il primo libro Sui poemi di Filodemo, P.Herc. 460), è segnalato da Janko (2000) 84. Vi si scorgono alcuni accenti (a quanto pare, posteriori alla copia del rotolo), che Janko attribuisce a un "neophyte practising accentuation on an old neglected tome”. 
del Carme segnalando talora delle pause ritmiche, talora dei punti in cui era necessario modulare in modo particolare la propria voce e farla crescere di intensità, al fine di dare un'interpretazione quanto più possibile esatta del testo". ${ }^{92}$

Questi elementi sono importanti che si voglia o meno dedurne che il P.Herc. 817 trasmette un testo “"vissuto', dunque letto e recitato ad alta voce perché noto”. Chiunque abbia apposto quei segni e qualunque siano stati i suoi intenti e i suoi interessi per il contenuto e il messaggio del poema, la loro presenza nel P.Herc. 817 costituisce una prova chiara che alcuni testi almeno della biblioteca erano letti, per ragioni che ci sfuggono, verso verso metà del I d.C.

\section{In conclusione}

Non vado oltre per evitare di dare l'impressione di affastellare ipotesi su ipotesi. Ho cercato di presentare in una forma per lo più aporetica i problemi che i nuovi elementi derivati dalla scoperta del libro $A b$ initio bellorum civilium di Seneca il vecchio hanno fatto emergere e con i quali dovranno, d'ora in poi, fare i conti coloro che si occuperanno dello studio della pars Latina della biblioteca di Ercolano.

Essa resta ancora la più misteriosa, ma la speranza che nuove scoperte aprano la via a ricerche che allargheranno le nostre conoscenze e la nostra sete di sapere non è forse vana. La decifrazione della subscriptio e dei sia pure magri resti del P.Herc. 1067 ne è una prova concreta che invita a continuare in questa direzione.

92 Scappaticcio (2008) 246 (da cui le citazioni che seguono). 
